\title{
Standounkt
}

Deutscher Nachhaltigkeitskodex

\section{Eine einseitige Erklärung ist kein Dialog}

\author{
Der Rat für Nachhaltige Entwicklung (RNE) hat einen Entwurf \\ zu einem Deutschen Nachhaltigkeitskodex für Unternehmen \\ vorgelegt. Die Stellungnahmen im Rahmen des Dialogverfah- \\ rens haben gezeigt, dass erhebliche Anpassungen des Entwurfs \\ notwendig sind. Von Rudolf X. Ruter
}

$D^{2}$ er vom Rat für Nachhaltige Entwicklung (RNE) vorgelegte Entwurf zu einem Deutschen Nachhaltigkeitskodex für Unternehmen hat unterschiedliche Reaktionen ausgelöst (1). An dem Dialogverfahren des RNE haben sich bis zum 25 . Februar 2011 insgesamt 74 Unternehmen, Organisationen, Institute und Einzelpersonen beteiligt (2).

\section{Inhaltliche Ergänzungen notwendig}

Die Schmalenbach-Gesellschaft für Betriebswirtschaft e.V. ist die einzige übergreifende betriebswirtschaftliche Vereinigung in Deutschland. Der Arbeitskreis „Nachhaltige Unternehmensführung“ (AKNU) untersucht, welche Instrumente Eigentümern, Geschäftsleitung, Bereichsleitung und Aufsichtsrat zur Verfügung stehen, um einzeln und gemeinsam verantwortlich $\mathrm{zu}$ handeln. Im folgenden stelle ich die Kernpunkte der Stellungnahme des AKNU vor (3).

- Dem bisherigen Entwurf fehlt es an einer klaren Definition bezüglich Inhalt und Umfang „Nachhaltigen Wirtschaftens“ (4). Gerade auch die internationale Diskussion, beispielsweise auf Ebene der Europäischen Union oder in der ISO-Norm 26000 werden nicht angesprochen.

- Unternehmen werden derzeit immer mehr belastet durch ausufernde Gesetze, Vorschriften, Empfehlungen und immer weitere Neuerungen. Beispielhaft dafür sind diverse Leitfäden zu verantwortlichem Handeln in der Wirtschaft, Einführung eines Ethikvorstands und ähnliches.

- Die Idee des Dialogs ist zu begrüßen, doch es wird bezweifelt, ob ein Kodex in Form einer einseitigen Erklärung einen Dialog beginnen kann.

- Derzeit bestehen schon zu viele untereinander nicht abgestimmte Kodizes parallel nebeneinander. Dazu gehören der Deutsche Corporate Governance Kodex, der Governance Kodex für Familienunternehmen, der Public Corporate Governance Kodex und weitere.

Grundsätzlich ist der AKNU gegen ein weiteres, separates „Empfehlungs-Regelwerk“. Die Idee der Nachhaltigkeit sollte vielmehr in bestehende Regularien weiter integriert und systematisiert werden. Eine Hilfestellung, die die Vielfalt der Anforderungen zusammenführt, ist hilfreich, jegliche Zusatzpflicht wirkt allerdings kontraproduktiv.

\section{Diskussion auf dem CSR Forum Deutschland}

Der AKNU empfiehlt, einen Kodex dergestalt $\mathrm{zu}$ formulieren, dass exakte messbare und nachprüfbare Ziele formuliert und kommuniziert werden können und dass er international als Vorlage verwendet werden kann.

Es sollte, wie beim Deutschen Corporate Governance Kodex, eine entsprechend legitimierte Regierungskommission mit einer ausreichenden Anzahl von
Vertretern der Wirtschaft unter Leitung der Wirtschaft eingerichtet werden.

Der AKNU moderierte auf einem der grössten jährlichen deutschen Nachhaltigkeitskongresse, dem 7. Deutschen CSR Forum, die Diskussions-Sektion „Brauchen wir einen Deutschen Nachhaltigkeitskodex“. Die Diskutanten kamen zu folgendem Ergebnis:

- Der RNE wird den Dialogprozess weiterführen. Wichtig ist dabei, zu einer besseren Handhabung und Übersichtlichkeit zu kommen.

- Es wird zügig vom RNE einen neuen Entwurf geben. Dabei sollten trotz Konzentration auf kapitalmarktorientierte Unternehmen auch klein- und mittelständische Unternehmen miteinbezogen werden.

- In einer weiteren Qualifizierungsphase werden vom RNE interessierte Unternehmen eingeladen, diesen neuen Entwurf weiterzuentwickeln.

- Rechtskonstruktionen zur Anwendung des Deutschen Nachhaltigkeitskodexes sollen in ihrer ganzen Breite, also unter Einschluss dessen, was heute bereits möglich ist, aufgeführt werden. Überlegungen zur Änderung des Aktiengesetzes werden dabei im nächsten Schritt zunächst zurückgestellt.

- Der RNE wird versuchen, bis September 2011 die Arbeit abzuschließen und einen Entwurf zu erstellen, der als Ratsempfehlung an die Bundesregierung adressiert werden kann.

\section{Anmerkungen}

(1) vgl. http://www.nachhaltigkeitsrat.de/ index.php?id $=6212$

(2) vgl. http://www.nachhaltigkeitsrat.de/projekte/ eigene-projekte/deutscher-nachhaltigkeitskodex/

(3) Entwicklung und vollständiger Text der Stellungnahme unter http://www.aknu.org/

(4) vgl. http://www.nachhaltigkeitsrat.de/ uploads/media/RNE_Nov_2010_-_Nachhaltigkeitskodex_Dialog_dt.pdf

I AUTOR + KONTAKT Rudolf X. Ruter ist Leiter des Arbeitskreises „Nachhaltige Unternehmensführung“ in der Schmalenbach-Gesellschaft für Betriebswirtschaft e.V.

Rudolf X. Ruter, Wirtschaftsprüfer und Steuerberater, Hauptmannsreute 44, 70192 Stuttgart. Tel.: +49 1752433028 , E-Mail: rudolf.x@ruter.de, Internet:www.ruter.de 


\section{Lizenzhinweis}

Die Beiträge in ÖkologischesWirtschaften werden unter der Creative-Commons-Lizenz "CC 4.0 Attribution Non-Commercial No Derivatives" veröffentlicht. Im Rahmen dieser Lizenz muss der Autor/Urheber stets genannt werden, das Werk darf nicht bearbeitet, abgewandelt oder in anderer Weise verändert und außerdem nicht kommerziell genutzt werden. Die digitale Version des Artikels bleibt für zwei Jahre Abonnent/innen vorbehalten und ist danach im Open Access verfügbar. 